\title{
Prediction of arrhythmia after intervention in children with atrial septal defect based on random forest
}

\author{
Hongxiao Sun ${ }^{1 \dagger}$, Yuhai $\mathrm{Liu}^{2 \dagger}$, Bo Song ${ }^{3^{*}}$, Xiaowen Cui ${ }^{1}$, Gang Luo ${ }^{1}$ and Silin Pan ${ }^{1 *}$
}

\begin{abstract}
Background: Using random forest to predict arrhythmia after intervention in children with atrial septal defect.

Methods: We constructed a prediction model of complications after interventional closure for children with atrial septal defect. The model was based on random forest, and it solved the need for postoperative arrhythmia risk prediction and assisted clinicians and patients' families to make preoperative decisions.

Results: Available risk prediction models provided patients with specific risk factor assessments, we used Synthetic Minority Oversampling Technique algorithm and random forest machine learning to propose a prediction model, and got a prediction accuracy of $94.65 \%$ and an Area Under Curve value of 0.8956 .

Conclusions: Our study was based on the model constructed by random forest, which can effectively predict the complications of arrhythmia after interventional closure in children with atrial septal defect.
\end{abstract}

Keywords: Atrial septal defect, Interventional therapy, Random forest, Synthetic Minority Oversampling Technique algorithm

\section{Background}

Atrial septal defect (ASD) is the common congenital heart disease (CHD), accounting for about $10 \%$ of the total CHD, including the following four types, primum, secundum, sinus venosus and unroofed coronary sinus types [1-3]. At present, interventional closure has become the first choice for the treatment of ASD, and the success rate is $97.9-98.7 \%$ [4-6]. The incidence of complications after interventional closure of ASD is 6.3$7.2 \%$, and arrhythmia is the most common complication $[6,7]$. For recent years, people's interest in artificial intelligence (AI) is increasing. Machine learning (ML), as a subset of $\mathrm{AI}$, is currently widely used in the medical

\footnotetext{
*Correspondence: 13789888999@163.com; silinpan@126.com

${ }^{\dagger}$ Hongxiao Sun and Yuhai Liu equal contribution.

${ }^{3}$ Qingdao University of Science and Technology, 266061 Qingdao, China

'Qingdao Women and Children's Hospital, Qingdao University, 266034 Qingdao, China

Full list of author information is available at the end of the article
}

field. ML can build models from dataset and makes predictions, helping experts make accurate predictions and assess disease risks in different environments, so as to develop personalized medical products to achieve precision medicine.

The onset of ASD and the occurrence of postoperative arrhythmias should not be ignored, the length of hospital stay (LOS) will increase, which will affect the healthcare system, especially with the current reduction in beds and increasement of costs [6]. Therefore, accurate prediction of the occurrence of arrhythmias will have a positive impact on health care indicators. Daghistani et al. [8] constructed a model for predicting the length of stay of patients with heart disease, and compared artificial neural networks, support vector machines, Bayesian networks and random forest classification algorithms. Based on the random forest model, the prediction performance was the best, specifically, the sensitivity, the accuracy and Area

C C The Author(s). 2021 Open Access This article is licensed under a Creative Commons Attribution 4.0 International License, which permits use, sharing, adaptation, distribution and reproduction in any medium or format, as long as you give appropriate credit to the original author(s) and the source, provide a link to the Creative Commons licence, and indicate if changes were made. The images or other third party material in this article are included in the article's Creative Commons licence, unless indicated otherwise in a credit line to the material. If material is not included in the article's Creative Commons licence and your intended use is not permitted by statutory regulation or exceeds the permitted use, you will need to obtain permission directly from the copyright holder. To view a copy of this licence, visit http://creativecommons.org/licenses/by/4.0/ The Creative Commons Public Domain Dedication waiver (http://creativecommons.org/publicdomain/zero/1.0/) applies to the data made available in this article, unless otherwise stated in a credit line to the data. 
Under Curve (AUC) were $80 \%, 80 \%$ and 0.94 , respectively. Przewlockakosmala et al. [9] based on ML, classified and predicted 177 heart failure with preserved ejection fraction patients and 51 asymptomatic control patients on account of rest and post-exercise echocardiography, and identified prognostic phenotypes among patients with heart failure and preserved ejection fraction. Tu et al. [10] built a neural network model based on 15 preoperative factors to predict the long-term hospital stay in ICU after adult cardiac surgery. Bhattacharya et al. [11] constructed an ensemble learning model based on logistic regression and Naïve Bayes to evaluate the risk of ventricular arrhythmia in patients with hypertrophic cardiomyopathy, and obtains a sensitivity, specificity and C-index of 0.73 , 0.76 and 0.83 , respectively. Alaa et al. [12] used neural networks, support vector machines, random forest, AdaBoost and gradient boosting to build predictive models to assess cardiovascular disease risks. Jalali et al. [13] constructed a deep neural network prediction model to improve the risk prediction of surgical outcome, and the accuracy and AUC value are 0.89 and 0.95 respectively. Luo et al. [14] used weighted support vector machine, weighted random forest and logistic regression to construct a predictive model for congenital heart defects. Based on nine comprehensive variables, weighted support vector machine has the best predictive performance, with the accuracy (ACC), Weighted accuracy (wtACC), AUC and G values of $0.9470,0.7681,0.8187$ and 0.8088 , respectively. Inspired by studies above, this paper proposes a random forest (RF) -based risk prediction model for arrhythmia after interventional closure in children with ASD.

\section{Methods}

\section{Datasets}

The dataset in this article is collected from the clinical dataset of children undergoing interventional closure of ASD at the Heart Center of Qingdao Women and Children's Hospital from July 2009 to June 2019. All family members of the children signed an informed consent form before the operation. There is a total of 269 children, including 96 males and 173 females. All the patients suffered secundum ASD, and the size of ASD patients with two or more ASD was added as the total defect size. There are 221 patients without arrhythmia, 40 postoperative complications with minor changes, and 8 postoperative complications with major changes. (Table 1) Minor changes includes 32 cases with new incomplete right bundle branch block, 6 cases with $\mathrm{I}^{\circ}$ atrial ventricular block (AV block) and 2 cases with premature atrial contractions. Major changes include 4 cases with frequent premature ventricular contractions, 3 cases with $\mathrm{II}^{\circ} \mathrm{AV}$ block and 1 case with accelerated junctional rhythm. Then, due to the imbalance between the data categories, the Synthetic Minority Oversampling Technique (SMOTE) algorithm is used to classify the data. (Table 2) Finally, the data is input into six classifiers to predict postoperative complications, and the prediction performance of the model is evaluated by the leave-oneout method. The comparison shows that the random forest as the classifier has the best prediction effect, with the prediction accuracy rate of $94.65 \%$ and the AUC value of 0.8956 .

\section{Synthetic minority oversampling technique algorithm}

SMOTE algorithm is proposed by Chawla et al. [15], which aims to synthesize some new positive samples to reduce category imbalance. It has been used in drugtarget interaction prediction research, protein posttranslational sites prediction research and extracellular matrix protein prediction research. The algorithm is briefly introduced as follows:

Given a positive sample $x$, search its nearest neighbor samples $k$, if the oversampling rate is $N$, then select the nearest neighbor sample $N$ from the $k$ nearest neighbor samples, denoted as $c_{1}, c_{2}, \ldots c_{N}$, then perform random linear interpolation $c_{1}, c_{2}, \ldots c_{N}$ between the positive samples $X$, and generate a new positive sample $P_{j}$ through Eq. (1):

Table 1 Demographic characteristics

\begin{tabular}{lllll}
\hline & Normal & minor changes & major changes & Total \\
\hline case number & 221 & 40 & 8 & 269 \\
Height $(\mathrm{m})$ & $0.94 \pm 0.18$ & $0.89 \pm 0.20$ & $1.10 \pm 0.34$ & $0.94 \pm 0.19$ \\
Weight $(\mathrm{kg})$ & $14.26 \pm 6.39$ & $13.28 \pm 8.16$ & $26.8 \pm 26.5$ & $14.49 \pm 8.14$ \\
CTR & $0.54 \pm 0.05$ & $0.55 \pm 0.05$ & $0.54 \pm 0.03$ & $0.54 \pm 0.05$ \\
ASD size $(\mathrm{mm})$ & $10.21 \pm 3.29$ & $10.44 \pm 3.00$ & $11.31 \pm 5.52$ & $10.28 \pm 3.32$ \\
LVEF $(\%)$ & $66.19 \pm 2.29$ & $65.98 \pm 2.47$ & $64.25 \pm 1.98$ & $66.10 \pm 2.33$ \\
BMI $\left(\mathrm{kg} / \mathrm{m}^{2}\right)$ & $15.59 \pm 1.83$ & $15.88 \pm 2.27$ & $17.63 \pm 5.04$ & $15.70 \pm 2.07$ \\
BSA $\left(\mathrm{m}^{2}\right)$ & $0.59 \pm 0.19$ & $0.55 \pm 0.22$ & $0.87 \pm 0.60$ & $0.59 \pm 0.22$ \\
Operating age (year) & $3.06 \pm 2.02$ & $2.66 \pm 2.60$ & $5.50 \pm 4.54$ & $3.07 \pm 2.25$ \\
\hline
\end{tabular}

CTR cardiothoracic ratio, LVEF left ventricular ejection fraction, $B M I$ body mass index, BSA Body surface area 
Table 2 Variables selected for machine learning modeling from the clinical datasets

\begin{tabular}{|c|c|c|c|}
\hline Input variable & Ranges & Input variable & Ranges \\
\hline$\overline{S e x^{a}}$ & $0-1$ & Potassium (mmol/L) & $3-5.7$ \\
\hline Height (m) & $0.64-1.30$ & Sodium (mmol/L) & $134-146$ \\
\hline Weight (kg) & $7-35$ & CK (U/L) & $13.83-276.87$ \\
\hline Lung blood ${ }^{a}$ & $0-1$ & CK-MB (U/L) & $8-43$ \\
\hline Precardiac space ${ }^{a}$ & $0-1$ & PT (S) & $9.8-13.7$ \\
\hline CTR & $0.4-0.65$ & INR & $0.8-1.19$ \\
\hline Right heart enlargement $^{a}$ & $0-1$ & APTT (S) & $20.1-31.8$ \\
\hline ASD size (mm) & $5-22$ & $\Pi \mathrm{T}(\mathrm{S})$ & 14-119.6 \\
\hline LVEF (\%) & $60-73$ & Fibrinogen (mg/dl) & $0.97-3.5$ \\
\hline Leukocyte $\left(\times 10^{9} / \mathrm{L}\right)$ & $4-13.88$ & $\mathrm{FDP}(\mu \mathrm{g} / \mathrm{ml})$ & $2.5-87.94$ \\
\hline Erythrocyte $\left(\times 10^{9} / \mathrm{L}\right)$ & $3.5-5.33$ & $\mathrm{D}$ dimer (mg/L) & $0-3.02$ \\
\hline Hemoglobin ( $\mathrm{g} / \mathrm{L}$ ) & 86-152 & $\mathrm{BMI}\left(\mathrm{kg} / \mathrm{m}^{2}\right)$ & $0.19-14.78$ \\
\hline Platelets $\left(\times 10^{9} / \mathrm{L}\right)$ & $100-443$ & $\mathrm{BSA}\left(\mathrm{m}^{2}\right)$ & $0.345-1.124$ \\
\hline Albumin (g/L) & $40-54.98$ & Operating age (year) & $0.50-14.78$ \\
\hline $\operatorname{ALT}(U / L)$ & $5.98-109.6$ & Creatinine ( $\mu \mathrm{mol} / \mathrm{l})$ & $4.78-318.69$ \\
\hline AST (U/L) & $10.21-95.17$ & Urea (mmol/L) & $0.99-7.34$ \\
\hline
\end{tabular}

CTR cardiothoracic ratio, LVEF left ventricular ejection fraction, BMI body mass index, BSA Body surface area, $A L T$ alanine transaminase, $A S T$ aspartate aminotransferase, INR international normalized ratio, $C K$ creatine kinase, $C K-M B$ creatine kinase-MB, $P T$ prothrombin time, $A P T T$ activated partial thromboplastin time, $\pi$ thrombin time, FDP fibrin degradation products

${ }^{a}$ categorical data. Sex: 0 female; 1 male. Lung blood: normal 0; increase 1; Precardiac space: normal 0; decrease 1. Right heart enlargement: false 0; true 1

$$
P_{j}=X+\operatorname{rand}(0,1) \times\left(c_{j}-X\right), J=1,2, \ldots N
$$

Among them, rand $(0,1)$ represents the random number in $(0,1)$.

SMOTE is an improved scheme based on the random oversampling algorithm. It is easy to have oversampling problem, which indicates that the information we gain from the model might be too specific, which is not general enough, owing to the fact that random oversampling algorithm is simply to copy samples to increase the minority samples. The basic idea of the Synthetic Minority Oversampling Technique algorithm is to analyze the minority samples and artificially synthesize new samples based on the minority samples and add them to the data set. Steps of the algorithm are as follows:

1. For each sample a in the minority class, use the Euclidean distance as the standard to calculate the distance from all samples in the minority class sample set to obtain its $k$ nearest neighbors.

2. Set a sampling ratio to determine the sampling magnification $N$ according to the sample imbalance ratio. For each minority sample $a$, randomly select several samples from its $k$ nearest neighbors, assuming that the selected nearest neighbor is $b$.

3. For each randomly selected neighbor $b$, construct a new sample with the original sample $a$ according to the following formula: $c=a+\operatorname{rand}(0,1) *|a-b|$.

\section{Random forest}

RF, proposed by Breiman [16], is an ensemble learning method based on decision tree classifiers, and has a wide range of applications in bioinformatics. The basic idea is that if there are $N$ samples with $M$ features in the original training set, RF selects $N$ samples from the original training set through Bootstrap resampling, and randomly selects $M$ features to train a fully grown tree. Repeat this process to obtain a set of decision tree combinations, summarize their outputs into the integrated model, and vote on the predicted value to generate the final prediction score of RF. Therefore, the number of decision trees and the randomly selected features are critical to build an accurate RF model.

\section{Support vector machine}

Support vector machine was first proposed by Cortes and Vapnik in 1995. It shows many unique advantages in solving small sample, nonlinear and high-dimensional pattern recognition, and can be extended to other machine learning problems such as function fitting.

The support vector machine method is based on the VC dimension theory of statistical learning theory and the principle of structural risk minimization. According to the limited sample information, the complexity of the model (that is, the learning accuracy of a specific training sample) and the learning ability (that is, error-free to find the best compromise between the ability to accurately identify any 
sample), in order to obtain the best promotion ability (or generalization ability).

\section{K-Nearest neighbor algorithm}

The K-Nearest Neighbor algorithm is one of the efficient and simplest methods for item classification [17]. In $\mathrm{KNN}$, training examples are expressed as points in the feature space in several separate classes. To predict the label of a new item $I x$, initially, it is projected in the problem feature space. Then, the distances between $I x$ and the $K$-nearest examples are calculated. Then, $I x$ is classified by a majority vote of its neighbors.

\section{Logistic regression}

Logistic model [18] can be applied to regression problems, and also can be used to solve classification problems. In the classification problem, the model can calculate the probability of belonging to each category according to a set of independent variables. Logistic regression model is the most widely used multivariate quantitative analysis method for binary dependent variable.

\section{AdaBoost}

AdaBoost (Adaptive Boosting) is a very popular boosting technique that aims at combining multiple weak classifiers to build one strong classifier. The original AdaBoost paper was authored by Yoav Freund and Robert Schapire.

A single classifier may not be able to accurately predict the class of an object, but when we group multiple weak classifiers with each one progressively learning from the others' wrongly classified objects, we can build one such strong model. The classifier mentioned here could be any of your basic classifiers, from Decision Trees (often the default) to Logistic Regression, etc.

\section{Decision tree}

Decision tree is one of the predictive modelling approaches used in statistics, data mining and machine learning.

Decision trees are constructed via an algorithmic approach that identifies ways to split a data set based on different conditions. It is one of the most widely used and practical methods for supervised learning. Decision Trees are a non-parametric supervised learning method used for both classification and regression tasks.

Tree models where the target variable can take a discrete set of values are called classification trees. Decision trees where the target variable can take continuous values (typically real numbers) are called regression trees. Classification and Regression Tree (CART) is general term for this.

\section{Model evaluation}

In statistical theory, the leave-one-out method, independent sample test and $K$-fold cross-validation are often used to evaluate the predicting performance of the model. The leave-one-out method directly divides the dataset into two mutually exclusive sets, one of which is used as the training set and the other as the test set. $K$ -fold cross-validation randomly divides the dataset into $K$ mutually exclusive subsets of similar size. Each time one of them is used as a test sample and $K-1$ are used as a training sample. The cross-validation process is repeated $K$ times, the average of $K$ times of crossvalidation is used as the prediction result of the classifier. In this paper, the leave-one-out method is used to train the model. In order to evaluate the prediction performance of the model, sensitivity, specificity, accuracy and Matthew's correlation coefficient are used as evaluation index, their definition are as follows:

$$
\begin{aligned}
S n & =\frac{T P}{T P+F N} \\
S p & =\frac{T N}{T N+F P} \\
A C C & =\frac{T P+T N}{T P+T N+F P+F N} \\
M C C & =\frac{T P \times T N-F P \times F N}{\sqrt{(T P+F N)(T P+F P)(T N+F P)(T N+F N)}}
\end{aligned}
$$

Among them, TP is the number of positive samples predicted to be correct, $F P$ is the number of negative samples predicted to be wrong, $T N$ is the number of negative samples predicted to be correct, and $F N$ is the number of positive samples predicted to be wrong. Sensitivity is the percentage of correct predictions for positive data, and specificity is the percentage of correct predictions for negative data. The value of Matthew's correlation coefficient (MCC) ranges from -1 to 1 , and the value of Sensitivity, specificity, and ACC ranges from 0 to 1 . In addition, Receiver Operating Characteristic is a curve based on the sensitivity and specificity, and AUC is the area under the Receiver Operating Characteristic (ROC) curve. As an indicator of the robustness of the prediction model, the closer the AUC value is to 1 , the better the prediction performance of the model is.

\section{Result}

Comparison of dataset imbalance processing methods

The dataset in this article includes 221 samples without complications and 48 samples with postoperative complications, including 40 cases of minor changes and 8 cases of major changes. The classification prediction performance may get a good overall classification accuracy, 
but be poor on the minority class samples. And the imbalance of the dataset often causes the prediction results to be biased towards the larger class. However, in many practical problems, the minority samples are more special and important. There is a serious data imbalance between the samples. In order to improve the generalization ability of the classifier and reduce the deviation caused by the imbalance of the dataset, before choosing the appropriate classifier, this article uses the SMOTE algorithm to process the samples to overcome the problem of the imbalance of the dataset. The feature vectors that have been balanced and unbalanced by the SMOTE algorithm are input into the random forest classifier, and the leave-one-out method is used to verify and compare the prediction results, as shown in Table 3.

It can be seen from Table 3 that for the dataset, the prediction model is constructed on the balanced dataset and the unbalanced dataset, and the obtained model evaluation indicators are quite different. In terms of the evaluation index accuracy, the accuracy obtained on the balanced dataset after Synthetic Minority Oversampling Technique processing has a greater advantage than the unbalanced dataset. However, due to the imbalance of the dataset itself, this indicator is used to measure. The pros and cons of the algorithm are not representative. After the dataset is balanced by the Synthetic Minority Oversampling Technique algorithm, the Area Under Curve value increases by $32.91 \%$. The Synthetic Minority Oversampling Technique algorithm balances the dataset by "synthesizing" the complication samples with minor changes and major changes samples, to increasing proportion in the dataset. Therefore, through the above comparative analysis, after Synthetic Minority Oversampling Technique processing, the prediction performance of the model is significantly improved.

It can be seen from Table 4 that for clinical data random forest algorithm as a predictive classification algorithm, the model has the best predictive performance, with accuracy, Sensitivity, specificity, Matthew's correlation coefficient and Area Under Curve reaching $94.65 \%, 92.50 \%, 94.98 \%, 0.7980$ and 0.8956 , respectively. The prediction accuracy, specificity, Matthew's correlation coefficient and Area Under Curve values are all higher than other classification algorithms. Using the logistic regression classifier, the model has the lowest prediction accuracy, with an accuracy of $78.60 \%$. The accuracy value of random forest is $16.05 \%, 15.72 \%$,
$12.04 \%, 9.70$ and $5.35 \%$ higher than logistic regression, K-Nearest Neighbor, decision tree, AdaBoost and Support Vector Machine respectively. The Matthew's correlation coefficient value and specificity value of the random forest classification algorithm are 12.06-37.49\% and $6.56-18.92 \%$ higher than the other five classification algorithms, respectively. From the evaluation indicators Sensitivity, specificity, accuracy and Matthew's correlation coefficient values, the random forest classifier achieves the best predictive performance.

\section{Comparison of prediction results of different machine learning algorithms}

In order to build an efficient prediction model, this paper selects six classification algorithms: logistic regression, $K$-Nearest Neighbor algorithm, decision tree, Support Vector Machine, AdaBoost, and RF to build the prediction model, selects the collected clinical information as the input feature vector, and uses the leave-oneout method to verify the evaluation. The prediction performance of the model and the prediction results of the dataset under different classifiers are shown in Table 4. In order to more intuitively analyze the prediction performance of different classifiers in the training dataset, draw the columns of the ACC value, MCC value and AUC value of the prediction model of complications after interventional closure of children with a ASD under six classifiers, as shown in Fig. 1. In addition, the ROC curve is used to compare the robustness of different prediction models. Figure 2 is the ROC curve obtained by the training set under the six classification algorithms.

It can be seen intuitively from Fig. 1 that the training dataset changes in the ACC, Sensitivity, specificity, MCC and AUC values of the six classifiers logistic regression, K-Nearest Neighbor, decision tree, AdaBoost, Support Vector Machine, and RF. For the ACC, the ACC varies from $78-94 \%$, the $\mathrm{MCC}$ value varies from 0.4 to 0.7 , and the AUC value varies from 0.72 to 0.89 . Both Support Vector Machine and RF classifiers achieve good AUC values for the training dataset

Choose the Receiver Operating Characteristic curve to compare the prediction performance of different classifiers. If the Receiver Operating Characteristic curve of one classifier is completely enclosed by the curve of another classifier, the prediction performance of the latter is better than the formable. It can be seen from Fig. 2

Table 3 Comparison of predict result with No-Synthetic Minority Oversampling Technique and Synthetic Minority Oversampling Technique method on dataset

\begin{tabular}{|c|c|c|c|c|c|}
\hline & Accuracy (\%) & Sensitivity (\%) & Specificity (\%) & MCC & AUC \\
\hline No-Synthetic Minority Oversampling Technique & 81.78 & 2.50 & 95.63 & -0.0335 & 0.5665 \\
\hline Synthetic Minority Oversampling Technique & 94.65 & 92.50 & 94.98 & 0.7980 & 0.8956 \\
\hline
\end{tabular}


Table 4 The prediction results of different classifiers

\begin{tabular}{|c|c|c|c|c|c|}
\hline Classifier & Accuracy (\%) & Sensitivity (\%) & Specificity (\%) & MCC & AUC \\
\hline Logistic Regression & 78.60 & 77.50 & 78.76 & 0.4231 & 0.7286 \\
\hline K-Nearest Neighbor & 78.93 & 97.50 & 76.06 & 0.5295 & 0.8219 \\
\hline Decision Tree & 82.61 & 80.00 & 83.01 & 0.4927 & 0.8035 \\
\hline AdaBoost & 84.95 & 87.50 & 84.56 & 0.5658 & 0.7489 \\
\hline Support Vector Machine & 89.30 & 95.00 & 88.42 & 0.6774 & 0.8744 \\
\hline Random Forest & 94.65 & 92.50 & 94.98 & 0.7980 & 0.8956 \\
\hline
\end{tabular}

that for the clinical dataset, the Receiver Operating Characteristic curve of Random forest completely includes the Receiver Operating Characteristic curve corresponding to the classifier's logistic regression, K-Nearest Neighbor, decision tree, AdaBoost and Support Vector Machine. Its Area Under Curve value is $16.70 \%, 7.45 \%, 7.37 \%, 9.20 \%$, 14.67 and $2.12 \%$ higher than logistic regression, KNearest Neighbor, decision tree, AdaBoost and Support Vector Machine respectively. In summary, Random forest's Receiver Operating Characteristic curve covers the largest area, indicating that the classification algorithm has the best predictive performance and robustness.

\section{Discussion}

ASD is the third common CHD, accounting for about $10 \%$ of the total incidence of CHD, of which about $70 \%$ are secundum ASD [1-3]. In 1948, Murray [19]closed the ASD under the condition of non-direct vision for the first time, opening the pioneering surgical treatment of ASD. At present, interventional close of ASD has gradually replaced surgery and become the preferred method for the treatment of ASD due to its advantages of less surgical trauma, high success rate, short hospitalization time and low cost. Intraoperative and postoperative arrhythmia is the most common complication of ASD intervention. Due to the stimulation of intracardiac structure by occluders and the push-pull test after the release of occluders, sinus tachycardia, atrial premature beats, atrial tachycardia, AV block and other arrhythmias may occur in some children during the operation. However, most of these children can relieve themselves after surgery, and a few children can last for hours or even weeks. After giving nutritional myocardial drugs or applying glucocorticoid and other treatments, most of them can restore sinus rhythm. Currently, it has been reported in

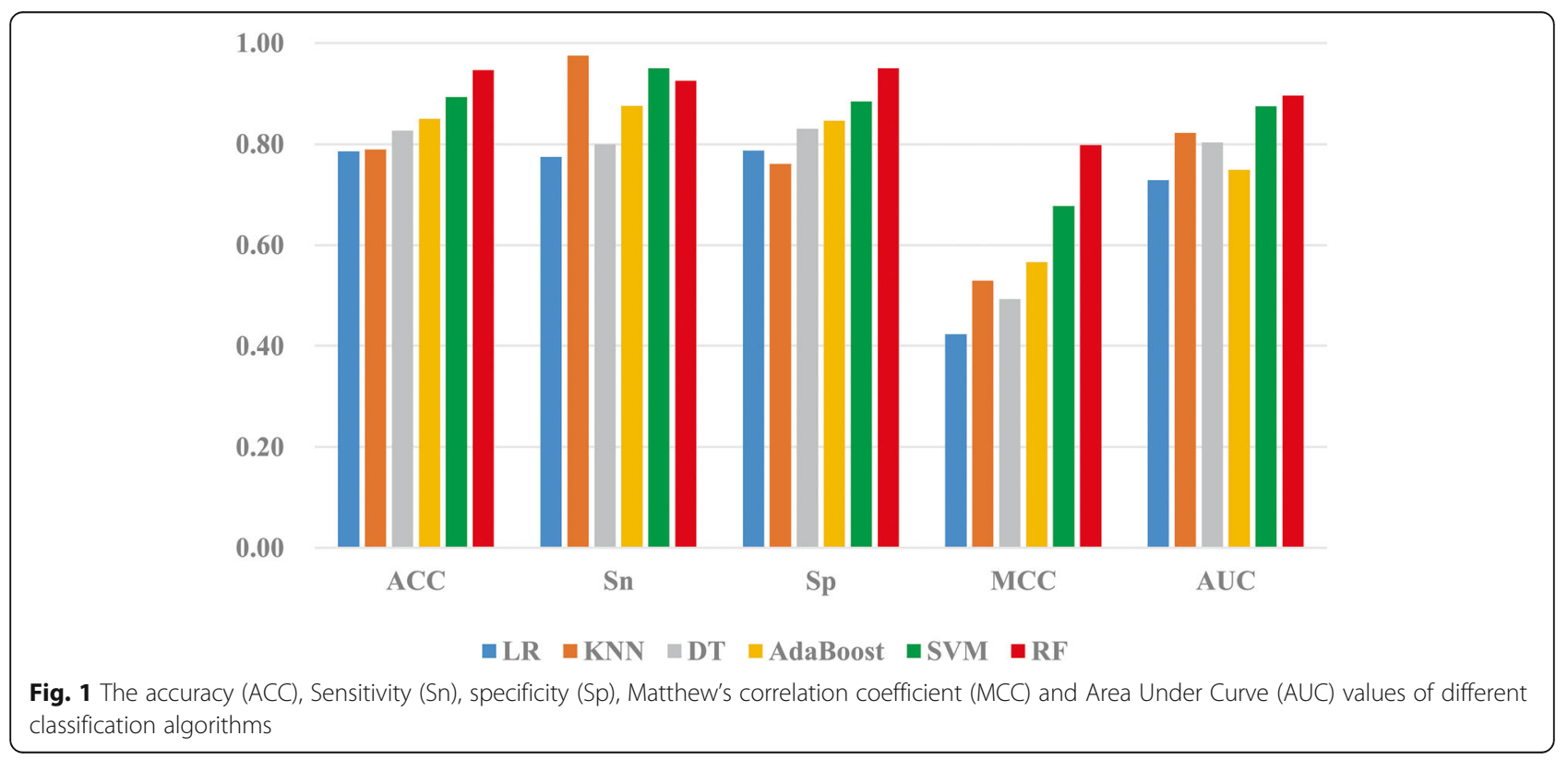




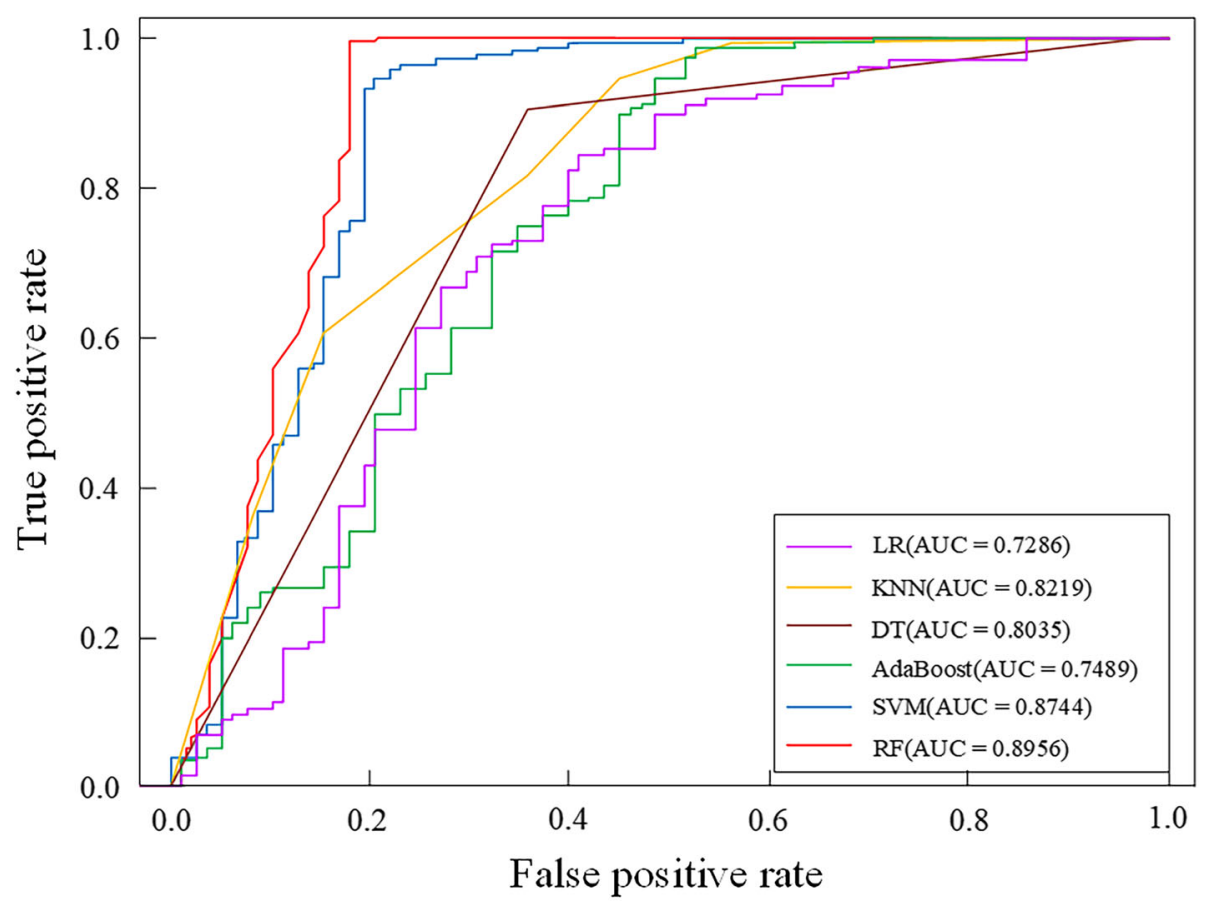

Fig. 2 The Receiver Operating Characteristic curves of different classification algorithms

paper that arrhythmias in children with ASD after intervention mainly include sinus bradycardia, sinus tachycardia, supraventricular tachycardia, atrial premature beats, ventricular premature beats, atrioventricular block, atrial fluttering and atrial fibrillation [20-23].

Komar et al. [22] believed that the diameter of the intraoperative occluder was closely related to the occurrence of arrhythmias after occlusion. Large diameter occluders are more likely to cause arrhythmias. ASD is adjacent to the Koch Triangle, and the atrioventricular node is located in the endocardium in front of the Koch Triangle, so it is easy to compress or damage the Koch Triangle by using a large occluder. Jin et al. [24] believed that when the ratio of occluder diameter to ASD size > 0.576 , the probability of arrhythmia was significantly increased. The size of ASD determines the type of occluder used intraoperatively. The larger the diameter of the occluder relative to the ASD, the higher the possibility that the edge of the occluder, especially the lower edge, will contact the Koch triangle, and thus the greater the possibility of injury to the Koch triangle. For children with low age, low body weight and large defects, the risk of postoperative arrhythmia is higher than other children due to the shorter length of atrial septum and larger diameter of occlusion device. During the intraoperative release of the occluder and at the early postoperative stage, the occluder may cause compression or friction damage to the surrounding tissues, resulting in corresponding tissue edema and damage, which will affect the conduction function of the electrical pathways of the surrounding heart tissues and cause AV block in the children. However, there are no studies that allow doctors to determine before surgery whether patients will develop postoperative arrhythmias, so as to prevent them early.

AI has become a symbol of the strategic core technology field since its emergence in the $1950 \mathrm{~s}$. In the $1970 \mathrm{~s}$, foreign scholars tried to create a computer algorithm to accurately identify pathological diagnosis [25], opening a new chapter of the rapid development of AI technology in the medical field. AI technologies such as ML can be trained to "learn" different features of data, quantify specific data or correlate with specific diseases [26], and even discover additional predictive information that may not be detectable by the naked eye [27]. Therefore, we constructed a model to prediction of arrhythmia after intervention in children with ASD based on random forest. Available risk prediction models provided patients with specific risk factor assessments, we used SMOKE algorithm and RF ML to propose a prediction model, and got a prediction accuracy of $94.65 \%$ and an AUC value of 0.8956 . This prediction model used all 32 variables in Table 2, and played a good role in assessing the risk of postoperative arrhythmias. Although there are many variables used in the model, these variables are all from routine preoperative examinations, and there is no need to add additional examination items, so the workload of clinicians and the economic burden of 
patients are not increased. Based on this model, we can carry out early prevention for patients with ASD who are at risk of developing postoperative arrhythmias, thus reducing the incidence of arrhythmias after ASD intervention and occlusion. At the same time, we will further screen variables in the following studies in order to obtain fewer and more accurate indicators to predict preoperative arrhythmias.

\section{Conclusions}

This article is based on the model constructed by random forest, which can effectively predict the complications of arrhythmia after interventional closure in children with atrial septal defect. Accurately predicting the risk of postoperative complications and their severity based on preoperative data will help to have more meaningful discussions with family members about the child after surgery. Through the practical application of this model, and after further verification with prospective dataset, we hope to improve clinical decision-making and provide the best predictive information for each family.

\section{Abbreviations}

ASD: Atrial Septal Defect; CHD: Congenital Heart Disease; Al: Artificial Intelligence; ML: Machine Learning; LOS: length of Hospital Stay; AUC: Area Under Curve; ACC: Accuracy; RF: Random Forest; AV Block: Atrial Ventricular Block; SMOTE: Synthetic Minority Oversampling Technique; CART: Classification and Regression Tree; MCC: Matthew's Correlation Coefficient; ROC: Receiver Operating Characteristic

\section{Acknowledgements}

Not applicable.

\section{Authors' contributions}

All authors contributed to the design or implementation of the study and approved the final manuscript as submitted. Liu, Sun and Cui drafted the manuscript. Liu and Cui provided an analysis of artificial intelligence. Sun and Luo had primary responsibility for patient screening, enrollment, outcome assessment. Song and Pan was responsible for study conception and design.

\section{Funding}

The work was financially supported by the National Natural Science Foundation of China (No. 81770316, 81970249), Qingdao Science and Technology Plan(20-3-4-47-nsh) and Shandong Taishan Scholarship (2018). The funding bodies only provides financial assistance, but does not provide assistance in the design of the study and collection, analysis, interpretation of data and in writing the manuscript.

\section{Availability of data and materials}

The datasets used and/or analyzed during the current study are available from the corresponding author on reasonable request.

\section{Declarations}

Ethics approval and consent to participate

The study was conducted in accordance with the Declaration of Helsinki and approved by the local ethics committee of Qingdao Women and Children's Hospital (No. QFELL-KY-2019-64). All participants' guardians signed the informed consent.

\section{Consent for publication}

Not applicable.

\section{Competing interests}

The authors declare that they have no competing interests.

\section{Author details}

'Qingdao Women and Children's Hospital, Qingdao University, 266034 Qingdao, China. ${ }^{2}$ Institute Oceanology, Chinese Academy of Sciences, 266071 Qingdao, China. ${ }^{3}$ Qingdao University of Science and Technology, 266061 Qingdao, China.

Received: 17 February 2021 Accepted: 27 May 2021

Published online: 16 June 2021

\section{References}

1. Botto LD, Correa A, Erickson JD. Racial and temporal variations in the prevalence of heart defects. Pediatrics. 2001;107(3):E32.

2. Le Gloan L, Legendre A, Iserin L, Ladouceur M. Pathophysiology and natural history of atrial septal defect. J Thorac Dis. 2018;10(Suppl 24):S2854-63.

3. Bradley EA, Zaidi AN. Atrial Septal Defect. Cardiol Clin. 2020;38(3):317-24.

4. Haas NA, Soetemann DB, Ates I, Baspinar O, Ditkivskyy I, Duke C, Godart F, Lorber A, Oliveira E, Onorato E, et al. Closure of Secundum Atrial Septal Defects by Using the Occlutech Occluder Devices in More Than 1300 Patients: The IRFACODE Project: A Retrospective Case Series. Catheter Cardio Inte. 2016;88(4):571-81.

5. Turner DR, Owada CY, Sang CJ, Jr., Khan M, Lim DS. Closure of Secundum Atrial Septal Defects With the AMPLATZER Septal Occluder: A Prospective, Multicenter, Post-Approval Study. Circ Cardiovasc Interv. 2017;10(8):e004212.

6. Villablanca PA, Briston DA, Rodes-Cabau J, Briceno DF, Rao G, Aljoudi M, Shah AM, Mohananey D, Gupta T, Makkiya M, et al. Treatment options for the closure of secundum atrial septal defects: A systematic review and meta-analysis. Int J Cardiol. 2017;241:149-55.

7. Du ZD, Hijazi ZM, Kleinman CS, Silverman NH, Larnitz K, Investigators A. Comparison between transcatheter and surgical closure of secundum atrial septal defect in children and adults - Results of a multicenter nonrandomized trial. J Am College Cardiol. 2002;39(11):1836-44.

8. Daghistani TA, Elshawi R, Sakr S, Ahmed AM, Al-Thwayee A, Al-Mallah MH. Predictors of in-hospital length of stay among cardiac patients: A machine learning approach. Int J Cardiol. 2019;288:140-7.

9. Przewlocka-Kosmala M, Marwick TH, Dabrowski A, Kosmala W. Contribution of Cardiovascular Reserve to Prognostic Categories of Heart Failure With Preserved Ejection Fraction: A Classification Based on Machine Learning. J Am Soc Echocardiogr. 2019;32(5):604-615 e606.

10. Tu JV, Guerriere MRJ. Use of a Neural-Network as a Predictive Instrument for Length of Stay in the Intensive-Care Unit Following Cardiac-Surgery. Comput Biomed Res. 1993;26(3):220-9.

11. Bhattacharya M, Lu DY, Kudchadkar SM, Greenland GV, Lingamaneni P, Corona-Villalobos CP, Guan YF, Marine JE, Olgin JE, Zimmerman S, et al. Identifying Ventricular Arrhythmias and Their Predictors by Applying Machine Learning Methods to Electronic Health Records in Patients With Hypertrophic Cardiomyopathy (HCM-VAr-Risk Model). Am J Cardiol. 2019; 123(10):1681-9.

12. Alaa AM, Bolton T, Di Angelantonio E, Rudd JHF, van der Schaar M. Cardiovascular disease risk prediction using automated machine learning: A prospective study of 423,604 UK Biobank participants. PLoS One. 2019;14(5): e0213653.

13. Jalali A, Lonsdale H, Do N, Peck J, Gupta M, Kutty S, Ghazarian SR, Jacobs JP, Rehman M, Ahumada LM. Deep Learning for Improved Risk Prediction in Surgical Outcomes. Sci Rep. 2020;10(1):9289.

14. Luo Y, Li Z, Guo H, Cao H, Song C, Guo X, Zhang Y. Predicting congenital heart defects: A comparison of three data mining methods. PLoS One. 2017; 12(5):e0177811.

15. Chawla NV, Bowyer KW, Hall LO, Kegelmeyer WP. SMOTE: Synthetic minority over-sampling technique. J Artif Intell Res. 2002;16:321-57.

16. Breiman L. Random forests. Mach Learn. 2001;45(1):5-32.

17. Ayyad SM, Saleh Al, Labib LM. Gene expression cancer classification using modified K-Nearest Neighbors technique. Biosystems. 2019;176:41-51.

18. Sun W. Research on risk control of personal credit loans of commercial banks based on logistic model. Zhejiang University; 2018.

19. MURRAY G. Closure of defects in cardiac septa. Ann Surg. 1948;8(6):843-52.

20. Al Akhfash AA, Al-Mesned A, Fayadh MA. Amplatzer septal occluder and atrioventricular block: A case report and literature review. J Saudi Heart Assoc. 2013;25(2):91-4. 
21. Chantepie A, Lefort B, Soule N, Bonnefoy R, Labarthe F. Atrioventricular block after transcatheter atrial septal defect closure using the Amplatzer septal occluder (R). Arch Pediatr. 2013;20(12):1333-6.

22. Komar M, Przewlocki T, Olszowska M, Sobien B, Stepniewski J, Podolec J, Mleczko S, Tomkiewicz-Pajak L, Zmudka K, Podolec P. Conduction Abnormality and Arrhythmia After Transcatheter Closure of Atrial Septal Defect. Circ J. 2014;78(10):2415-21.

23. Yamamoto T, Kanazawa H, Tanosaki S, Goto S, Kimura M, Tsuruta H, Itabashi Y, Murata M, Kunitomi A, Aizawa Y, et al. A Novel Mechanism of Atrioventricular Block Following Transcatheter Closure of an Atrial Septal Defect. JACC Cardiovasc Interv. 2016;9(19):2067-9.

24. Jin $M$, Ding WH, Wang XF, Guo BJ, Liang YM, Xiao YY, Sun CF. Value of the Ratio of Occluder Versus Atrial Septal Length for Predicting Arrhythmia Occurrence after Transcatheter Closure in Children with Ostium Secundum Atrial Septal Defect. Chin Med J (Engl). 2015;128(12):1574-8.

25. Perez JE, Waggoner AD, Barzilai B, Melton HE Jr., Miller JG, Sobel BE. On-line assessment of ventricular function by automatic boundary detection and ultrasonic backscatter imaging. J Am Coll Cardiol. 1992;19(2):313-20.

26. Lee JG, Jun S, Cho YW, Lee H, Kim GB, Seo JB, Kim N. Deep Learning in Medical Imaging: General Overview. Korean J Radiol. 2017;18(4):570-84.

27. Madani A, Arnaout R, Mofrad M, Arnaout R. Fast and accurate view classification of echocardiograms using deep learning. NPJ Digit Med. 2018;1:6.

\section{Publisher's Note}

Springer Nature remains neutral with regard to jurisdictional claims in published maps and institutional affiliations.

Ready to submit your research? Choose BMC and benefit from:

- fast, convenient online submission

- thorough peer review by experienced researchers in your field

- rapid publication on acceptance

- support for research data, including large and complex data types

- gold Open Access which fosters wider collaboration and increased citations

- maximum visibility for your research: over $100 \mathrm{M}$ website views per year

At $\mathrm{BMC}$, research is always in progress.

Learn more biomedcentral.com/submissions 